\title{
On the importance of recrystallization to reproduce the Taylor impact specimen shape of a pure nickel
}

\author{
Hervé Couque ${ }^{\mathrm{a}}$ \\ Nexter Munitions, 7 route de Guerry, 18023 Bourges, France
}

\begin{abstract}
Taylor tests are a mean to investigate the dynamic plastic and failure behaviour of metals under compression. By taking in account the strengthening occurring at high strain rates, the Taylor final diameter of a pure nickel impacted at $453 \mathrm{~m} / \mathrm{s}$ have been numerically reproduced by $13 \%$. Through post-mortem observations of the specimen impacted at $453 \mathrm{~m} / \mathrm{s}$, a recrystallization process has been found to occur resulting in a softening of the pure nickel. Subsequent numerical simulations taking in account this softening have been found to reduce the difference between experimental and numerical diameter by $10 \%$.
\end{abstract}

\section{Introduction}

There are known challenges with the numerical simulation of large plastic deformation of metals generated under high strain rates [1]. One of the challenges is the stabilization of plastic deformation when the viscous behavior of the dislocations prevailed. Such challenge has been answered by taking in account the strengthening occurring in the viscous regime with the use of adequate constitutive models such as the Zerrilli Amstrong or modified JohnsonCook models [2]. However, a challenge remains with regard to the accuracy of the simulations. This challenge has been quantified with the numerical simulations of symmetric Taylor tests conducted with a pure nickel. At an impact speed of $453 \mathrm{~m} / \mathrm{s}$, the numerical simulation was found to underestimate the final diameter of the Taylor specimen by $13 \%$ [2].

Recent post-mortem analyses of pure nickel compression specimens tested in the viscous regime reveal the occurrence of recovery/ recrystallization [3]. In this work the recrystallized nickel was found to have a similar dislocation density and strength that the initial state of the nickel.

These results have motivated to reconsider the Taylor test conducted at an impact speed of $453 \mathrm{~m} / \mathrm{s}$.

In a first step, post-mortem analyses of the Taylor specimen have been conducted to quantify ductile failure occurring in the center of the specimen and to provide recrystallization evidences based on hardness measurements. In a second step, numerical simulations have been conducted with a tensile failure criterion and a modified Johnson-Cook model taking in account the softening when recrystallization occurred.

\section{The symmetric taylor test}

The Taylor test is a mean to validate constitutive models at strain rate ranging from $10^{3}$ to $10^{4} \mathrm{~s}^{-1}$ and

\footnotetext{
${ }^{a}$ Corresponding author: h.couque@nexter-group.fr
}

to evaluate dynamic compression failure properties of materials. Taylor testing has been intensively used in the original configuration consisting of one cylindrical specimen impacting an anvil made of hard steel as to reproduce a rigid wall, referred as the single Taylor test $[4,5]$. To simulate the single Taylor test it is required to make an estimation of the friction coefficient between the specimen and the anvil, as well as, to assume that the anvil is rigid.

With the symmetric loading procedure introduced by Erlich et al. [6], such hypotheses are no longer needed. When compared to single Taylor testing, symmetric Taylor testing involves one cylindrical specimen sent with a gas gun against another identical specimen located at the gun muzzle. For the symmetric Taylor test, it is equivalent either to send two specimens against a second specimen at rest. Evidences of the importance of friction have been revealed in a comparison between the two Taylor test configurations [7].

One inconvenient of Erlich's approach is the use of ceramic pins to locate the specimen sitting at the gun muzzle making difficult coaxial and planar impact of the two specimens. To facilitate planar and coaxial impacts, two testing procedures involving Teflon sabots have been developed by the author [8]. The first procedure addresses materials of strength greater than $800 \mathrm{MPa}$ in which the Teflon sabots are deformed with the Taylor specimens. The second procedure was developed for low strength materials, 100-800 MPa, in which a Teflon sabot and Teflon supports are designed to not interfere with the specimens. It is the last procedure that was employed for the pure nickel impacted at $453 \mathrm{~m} / \mathrm{s}$.

\subsection{Pure nickel}

A nickel elaborated by Eramet in plate $12 \mathrm{~mm}$ thick with a $99,99 \%$ content of nickel was employed. The nickel was used in a heat treated condition providing a quasi-static yield stress of $240 \mathrm{MPa}$ [2].

This is an Open Access article distributed under the terms of the Creative Commons Attribution License 4.0, which permits unrestricted use, distribution, and reproduction in any medium, provided the original work is properly cited. 


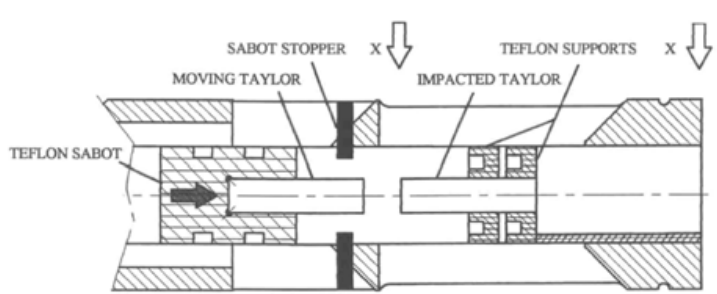

Figure 1. Schematic of the symmetric Taylor test for low strength materials.

\subsection{Experimental procedure}

The experimental set-up is shown in Fig. 1 with two Taylor specimens $9 \mathrm{~mm}$ in diameter and $36 \mathrm{~mm}$ in length. The loading system consist of a $25 \mathrm{~mm}$ caliber gas gun launching one Taylor specimen guided with a Teflon sabot which is arrested prior impact using copper inserts. The velocity of the launched specimen was measured using two laser beams separated by $18 \mathrm{~mm}$ located $30 \mathrm{~mm}$ behind the impact plane. The Taylor specimen located at the gun muzzle is positioned with two crushable Teflon supports. $\mathrm{X}$ ray observations were conducted with a Teflon witness bar in contact with the second Teflon support as to verify that the Teflon supports of the impacted Taylor specimen did not move prior impact [8].

A numerical investigation has been conducted to establish that the Teflon supports did not interfere during the plastic deformation of the Taylor specimens made of aluminium for a test conducted at an impact speed of $335 \mathrm{~m} / \mathrm{s}$ [8]. The equivalent plastic strain profiles of the Taylor specimens are provided when passing the Teflon supports and after passing the Teflon supports, see Figs. $2 \mathrm{a}$ and $2 \mathrm{~b}$. The strain profiles remain unchanged confirming that the stress applied by the Teflon supports on the Taylor specimens remains lower than the yield stress. This is verified with the von Mises and equivalent plastic strain records of the two material points I and II located in the plastic deformed region of the impacted Taylor specimens, see Figs. 2c and 2d.

\section{Results}

Previous numerical results obtained with modified Johnson-Cook models are first reported. Then, results from post-mortem analyses of the Taylor specimen impacted at $453 \mathrm{~m} / \mathrm{s}$ are provided revealing damage in the impact region composed of a tensile failure zone and a recrystallization zone. Finally, numerical results taking in account these two damage phenomena are reported.

\subsection{Numerical results with a modified Johnson-Cook model}

Numerical simulations of the Taylor test were conducted with a modified Johnson-Cook model (JCM) taking in account the strengthening occurring in the viscous regime [2]. The simulations were conducted with AUTODYN with an optimized numerical model of the Taylor specimen composed of cells $100 \mu \mathrm{m}$ in size in the plastically loaded region and $300 \mu \mathrm{m}$ in size for the elastically loaded region. The modified Johnson-Cook model includes a power strain a)

b)
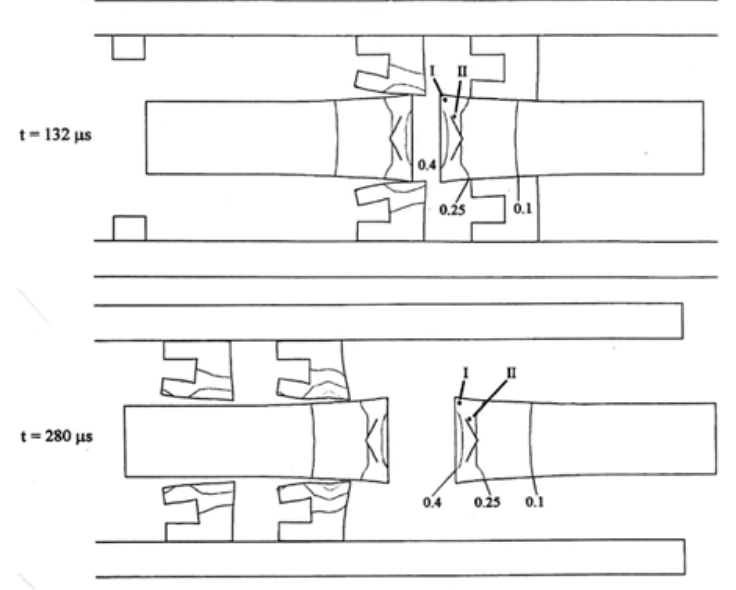

c)

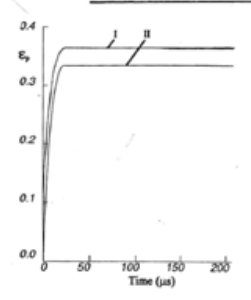

d)

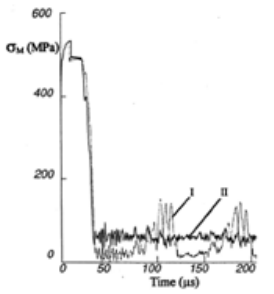

Figure 2. Numerical results of Taylor specimens made of aluminium tested at an impact speed of $335 \mathrm{~m} / \mathrm{s}$ : a) and b) equivalent strain profiles at 132 and $280 \mu$ s, respectively; c) and d) von Mises stress history and equivalent plastic strain history of material points I and II, respectively.

rate component added to the logarithm strain rate term of the original Johnson-Cook (JC) formulation, $\left.D(\varepsilon) / \varepsilon_{j}\right)^{k}$, with $D$ and $k$ two constants. This component is normalized by a reference strain rate, $\varepsilon_{1}$, equal to $10^{3} \mathrm{~s}^{-1}$ delimitating the thermally activated regime and the viscous regime. The equivalent stress function of plastic strain, $\varepsilon_{p}$, strain rate, $\varepsilon$ and temperature, $T$, is expressed as:

$$
\begin{aligned}
\sigma= & \left(A+B \varepsilon_{p}^{n}\right)\left(1+C \ln \left(\varepsilon^{\prime} / \dot{\varepsilon}_{o}\right)+D\left(\varepsilon^{\prime} / \dot{\varepsilon}_{1}\right)^{k}\right) \\
& \times\left(1-\left[\left(T-T_{r}\right) /\left(T_{m}-T_{r}\right)\right]^{m}\right)
\end{aligned}
$$

with the usual constants of the classical Johnson-Cook formulation: $T_{r}, T_{m}$ the room and melting temperatures, $\varepsilon_{o}$, a reference strain rate equal to $1 \mathrm{~s}^{-1}$ and $A, B, C$, $n, m$ five material constants describing the mechanical response. This formulation enables to go back to the classical Johnson-Cook formulation when strain rates are lower than $10^{3} \mathrm{~s}^{-1}$.

At low impact speeds, numerical simulations of the final diameter of the Taylor specimens were improved with the use of the JCM model when compared to the JC model with difference less than $10 \%$, see Fig. 3. In an another hand, numerical simulations at high impact speeds were found to provide a significant difference, with an underestimation of the final diameter by $17 \%$ for the test conducted at $453 \mathrm{~m} / \mathrm{s}$.

To improve the numerical simulation at high impact speeds, a saturation stress in the $10^{4} \mathrm{~s}^{-1}$ strain rate regime was introduced based on Steinberg's argument which stipulates that at a given strain rate all effects of strain rate have saturated and the material strength becomes independent of strain rate [9]. Strength levelling observed 


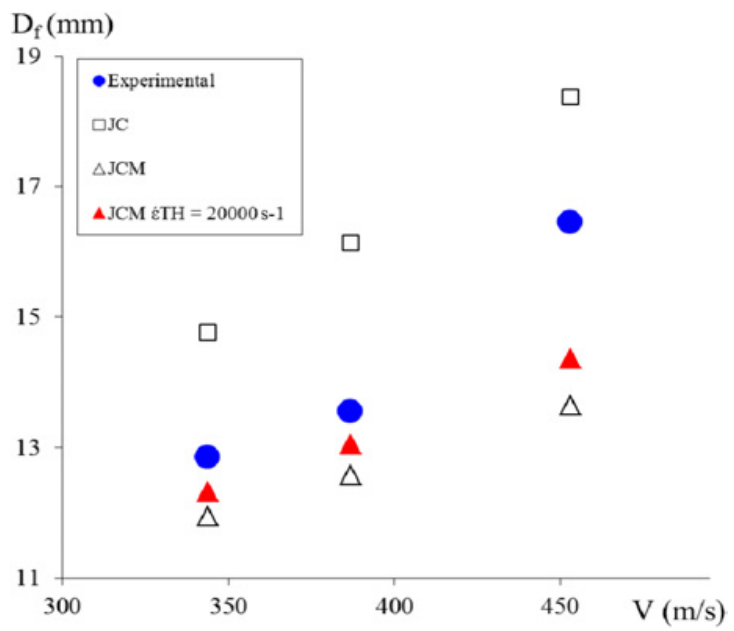

Figure 3. Experimental and numerical final diameter of the Taylor specimens versus impact speed. Numerical diameters were generated using three materials models: Johnson-Cook classical formulation (JC), Modified Johnson-Cook model (JCM), Modified Johnson-Cook model with a strain rate threshold, $\dot{\varepsilon}_{T H}$, of $20000 \mathrm{~s}^{-1}$.

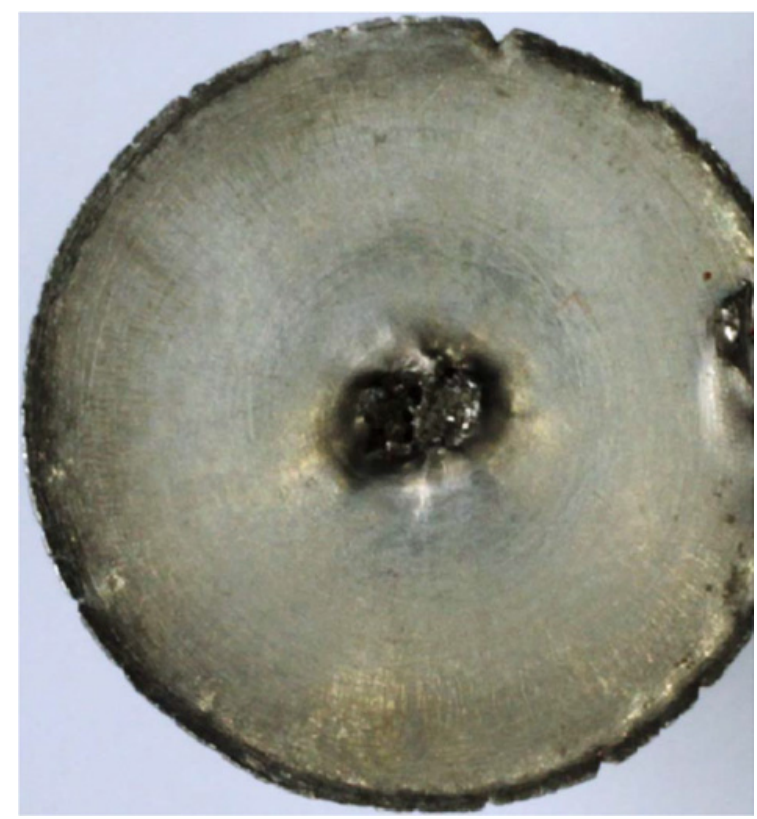

Figure 4. Tensile failure zone of the Taylor specimen tested at $453 \mathrm{~m} / \mathrm{s}$. The final diameter of the specimen is $16,45 \mathrm{~mm}$.

at high impact speeds with the compression test has motivated the choice of a strain rate threshold of $20000 \mathrm{~s}^{-1}$ [2]. The use of the JCM model with the strain rate threshold provides a better fit at high impact speeds with an underestimation of the Taylor final diameter reduced to $13 \%$ for the test conducted at $453 \mathrm{~m} / \mathrm{s}$, see Fig. 3 .

\subsection{Post-mortem analyses}

Figure 4 reveals a tensile failure zone in the center of the impact faces of the Taylor specimens, 2,5 $\mathrm{mm}$ in size. This

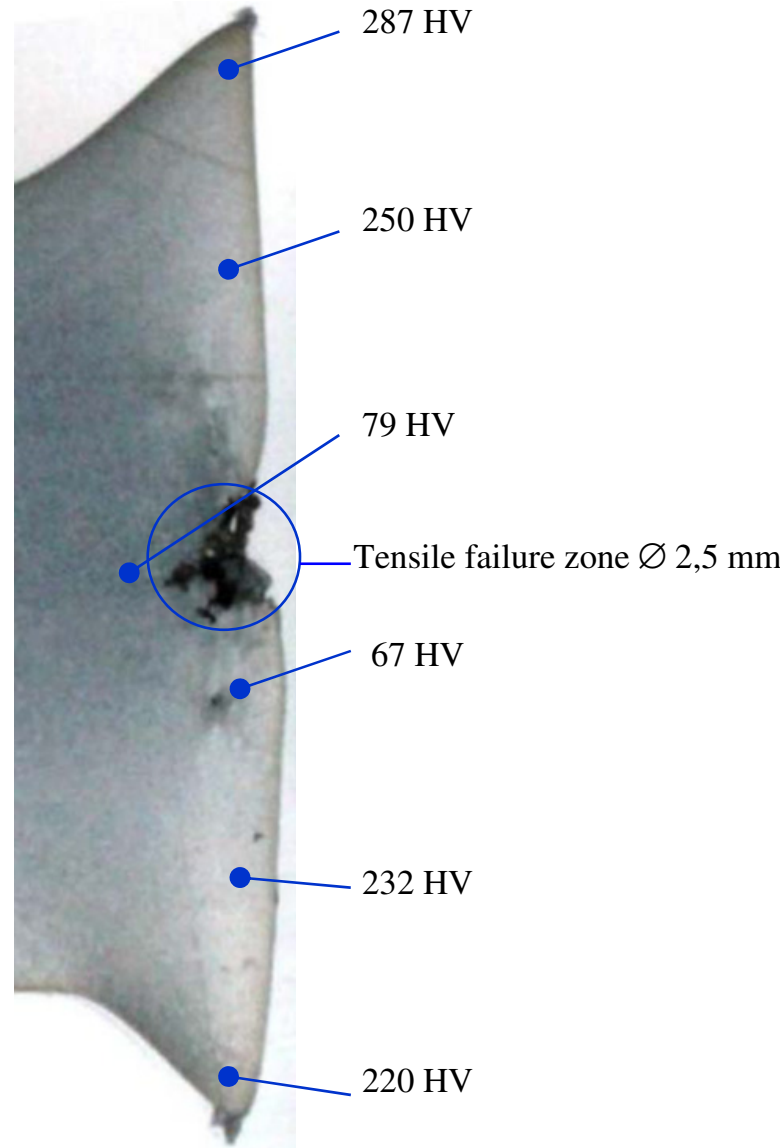

Figure 5. Sectioned Taylor specimen revealing the tensile failure zone and the variation of the hardness.

zone extends inside the specimen to about 2,5 $\mathrm{mm}$ in depth, see Fig. 5.

Vickers hardness measurements were generated in the impact zone with an applied force of 0,005 kgf, see Fig. 5. The hardness of the initial nickel was measured to be 179 . A decrease of the hardness to 67 was measured near the tensile failure zone, see Fig. 6. Such low hardness confirms that recrystallization occurred. Based on a yield stress of $240 \mathrm{MPa}$ for the initial nickel, the yield stress associated with the recrystallized nickel was estimated to be of the order of $50 \mathrm{MPa}$.

\subsection{Numerical results with a tensile failure criterion}

Numerical simulations were conducted with a failure strain criterion applied to the principal strain $\varepsilon_{11}, \varepsilon_{22}$ and $\varepsilon_{33}$, see Fig. 6a. A failure criterion of 0,63 has to be applied to reach the planar size of tensile failure zone, $\mathrm{D}_{\mathrm{tf}}$, of $2,5 \mathrm{~mm}$, see Fig. 5. For this failure criterion, no significant increase of the Taylor final diameter has been noticed, see Fig. 6 b.

The failure zone was not quite reproduce as it was limitated to a tensile failure of $1 \mathrm{~mm}$ in depth. Based on the work of Chapman and all, the extend of tensile failure inside the specimen comes from the coalescence of radial release waves at the specimen axis [10]. As suggested in this work, simulations conducted with a critical treshold 


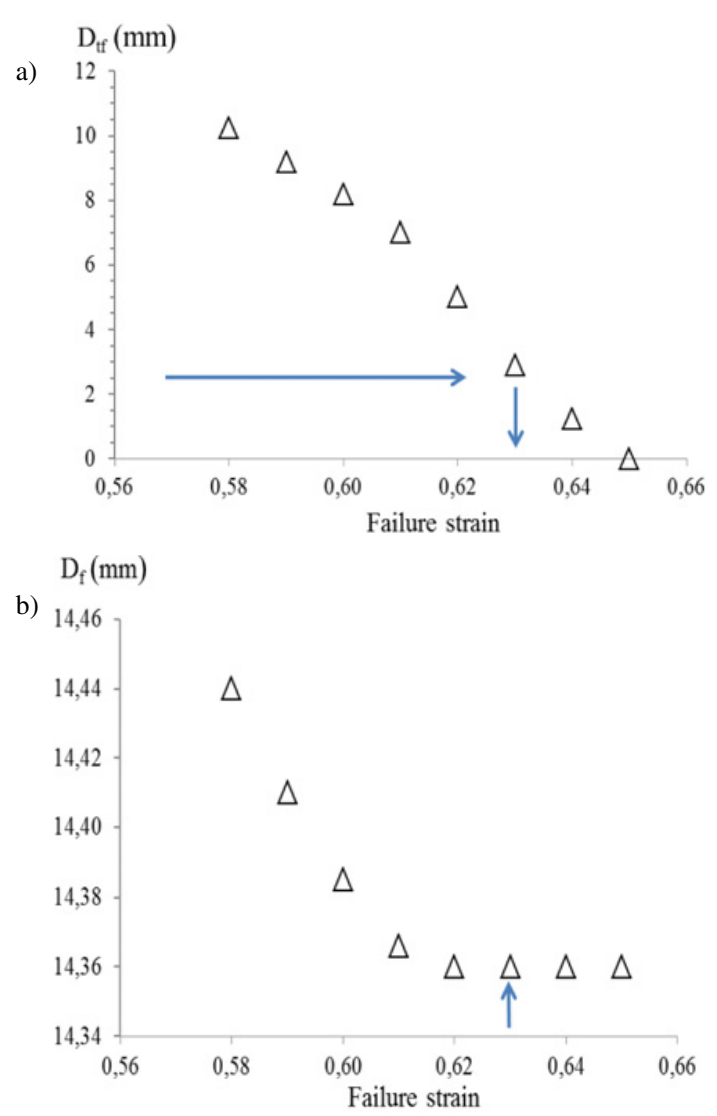

Figure 6. Tensile failure: a) planar size function of the failure criterion, b) Taylor final diameter function of the failure criterion.

pressure appear to be a mean to generate this bulk tensile failure.

However, the ability to reproduce the radial tensile failure demonstrates that the difference between the experimental and numerical diameters does not originate from the failure process occuring in the Taylor specimen.

\subsection{Numerical results with a recrystallization criterion}

The decrease in strength associated with the recrystallization phenomena was taken in account in the numerical simulation by introducing a recrystallization temperature criterion. The work of Lichtenberger provides recrystallized hardness temperature curves for different level of initial plastic deformation [11]. From these curves, quasi-static recrystallization temperatures of 290 and $230^{\circ} \mathrm{C}$ are deduced for a plastic deformation of 30 and $70 \%$, respectively. Based on this work, a temperature of $230^{\circ} \mathrm{C}$ was selected as a recrystallization temperature criterion. Such temperature is reached $12 \mu \mathrm{s}$ after impact, see Fig. 7. At this instant the plastic deformation is about $80 \%$.

Numerical simulations were consequently generated incorporated two type of JCM models, with the first model representative of the original material, see Sect. 3.1, and the second model representative of the recrystallized material. The yield and hardening coefficients for the
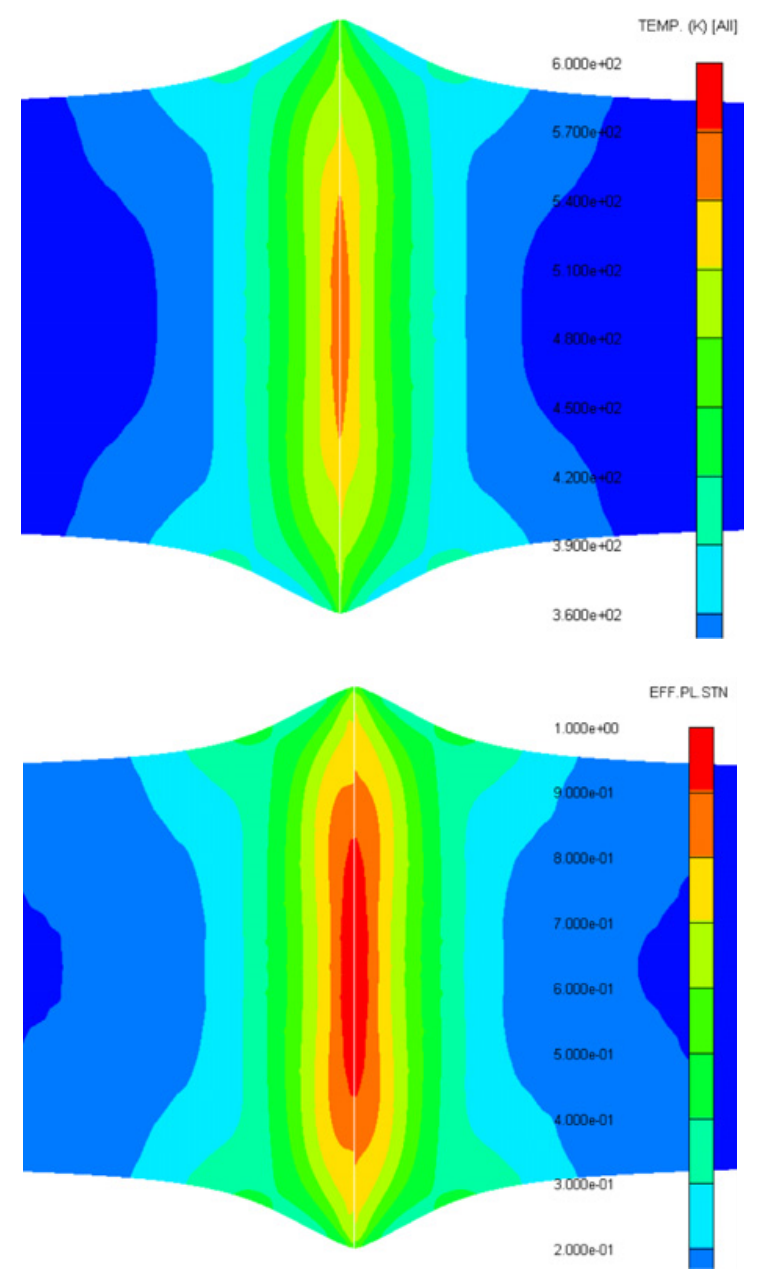

Figure 7. Temperature and effective plastic strain at $12 \mu \mathrm{s}$ after impact.

recrystallized material were identified as to provide a rigid plastic behaviour with a quasi-static yield stress of $50 \mathrm{MPa}$. Precisely, the A and B coefficients of the JCM models were 200 and $800 \mathrm{MPa}$ for the first model, and 100 and $400 \mathrm{MPa}$ for the second model, respectively. The simulations were conducted by monitoring the temperature. At the recrystallization temperature criterion of $230^{\circ} \mathrm{C}$, the model representative of the recrystallized material was applied.

Results conducted with these models are provided in Fig. 8. To describe the difference in final diameter, a simulation was conducted with one specimen without recrystallization criterion (a2) and the other with a recrystallization criterion (a1). A larger final diameter was generated with the specimen incorporating the recrystallization criterion which is less than $10 \%$ the experimental diameter, see Fig. 9. The same diameter was obtained when the simulation of the two Taylor specimens involving the recrystallization temperature criterion, see Fig. 8 b.

These results provide a framework to measure the dynamic recrystallization temperature of metals. Further investigations are required to improve the accuracy of the numerical data. These investigations will have to provide 
a1)

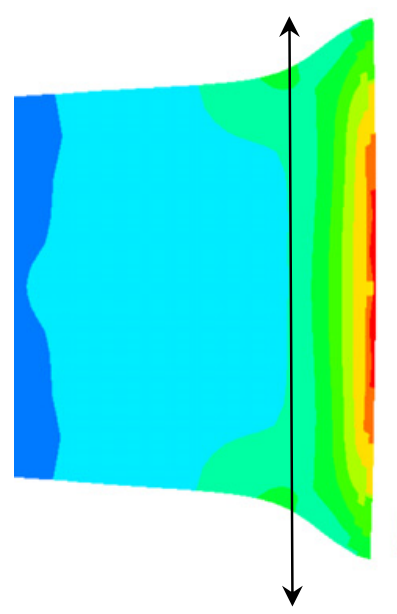

b)

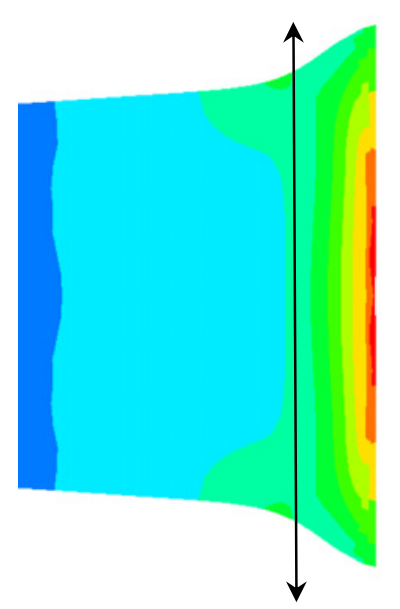

a2)

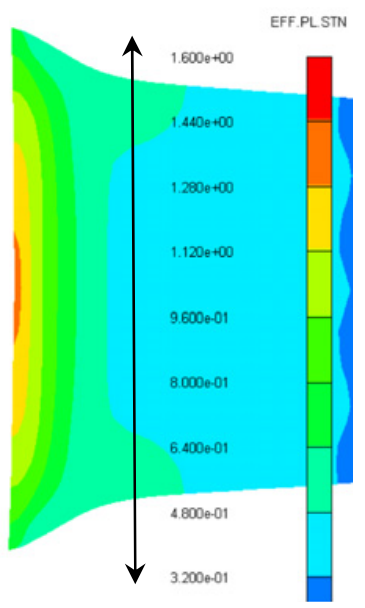

$\mathrm{D}_{\mathrm{f}}=14,2 \mathrm{~mm}$

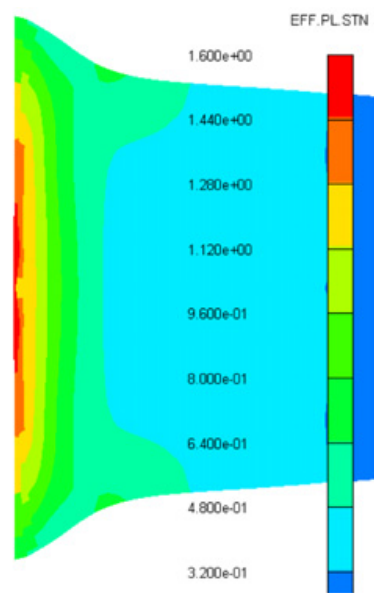

Figure 8. Final effective plastic strain maps with JCM models without a recrystallization criterion (a2) and with a recrystallization criterion (a1) and (b). The numerical diameters are indicated. The experimental diameter is $16,4 \mathrm{~mm}$.

more precise data on the size of the recrystallized zone and on the mechanical responses of the recrystallized material.

\section{Conclusion}

Taylor tests have been found to be a mean to investigate the dynamic deformation of a pure nickel in the $10^{3}-10^{4} \mathrm{~s}^{-1}$ strain rate regime. Numerical experimental investigations taking in account the strengthening occurring in this regime have been a mean to reproduce the Taylor final diameter by $7 \%$ when considering an impact speed of $350 \mathrm{~m} / \mathrm{s}$. However at higher impact speeds, significant difference between numerical and experimental diameters remained, up to $13 \%$ for an impact speed of $453 \mathrm{~m} / \mathrm{s}$. Through post-mortem observations of the specimen impacted at $453 \mathrm{~m} / \mathrm{s}$, a recrystallization process has been found to occur. Such recrystallization process results in a softening of the pure nickel. Subsequent

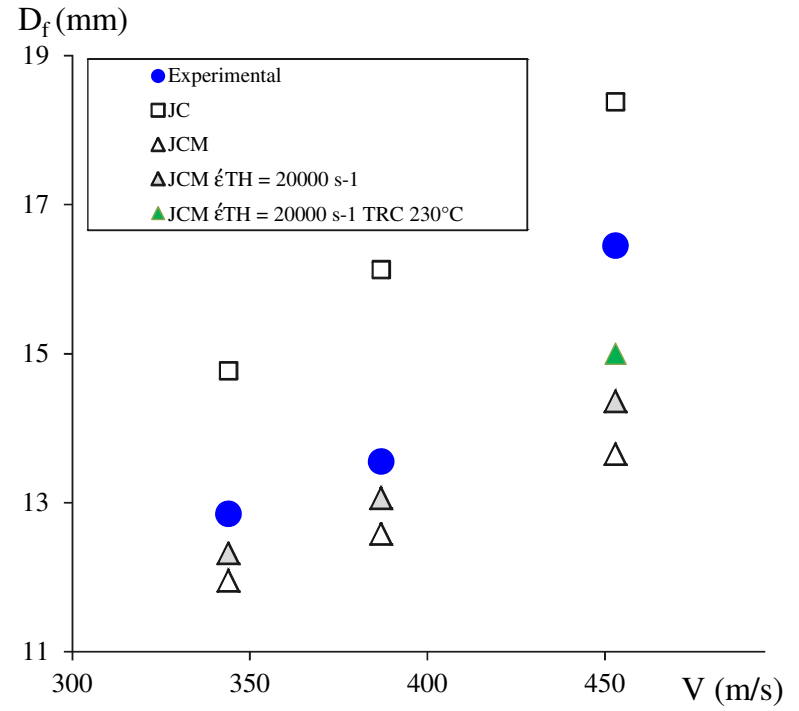

Figure 9. Experimental and numerical final diameter of the Taylor specimens versus impact speed incorporating the data generated with the recrystallization temperature criterion of $230^{\circ} \mathrm{C}$.

numerical simulations taking in account this softening have been found to be a mean to reduce the difference between experimental and numerical final diameters from $13 \%$ to $10 \%$.

The author thanks M. Jean-Charles Saint Supery from Nexter Systems for generating the hardness measurements.

\section{References}

[1] H. Couque, Phil. Trans. R. Soc. A 372, 218 (2014).

[2] H. Couque, R. Boulanger, $23^{\text {rd }}$ Int. Symp. Ballistics, Eds F. Galvez \& V. Sanchez-Galvez, 255 (2007).

[3] G. Dirras, H. Couque, J. Gubicza, A. Ouarem, T. Chauveau, P. Jenei, Mat. Sc. \& Eng. 1 527, 4128 (2010).

[4] G. Taylor, Proc. Roy. Soc. London A 194, 289 (1948).

[5] S. Jones, J. Drinkard, W. Rule, L. Wilson, Int. J. Imp. Engng 21, 1 (1998).

[6] D. Erlich, D. Shockey, L. Seaman, Conf. Shock Waves Condensed Matter, Eds Amer. Inst. Phys. 402 (1982).

[7] H. Couque, A. Lichtenberger, E. Lach, B. Salesse J. Phys; IV France 10, 353 (2000).

[8] H. Couque J. Phys; IV France 10, 179 (2000).

[9] D. Steinberg, S. Cochran S. G., M. Guinan, J. Appl. Phys., 561, 1498 (1980).

[10] D. Chapman, D. Radford, P. Church, Int. Jr. Fr. 134, 41 (2005).

[11] A. Lichtenbeger, ISL Technical Report "Etude du cuivre et du nickel pour revêtements de charges creuses mise en forme par matriçage", S-R 902/90 (1990). 